\title{
Plane strain deformation of an orthotropic elastic medium using an eigenvalue approach
}

\author{
N. R. Garg ${ }^{1}$, Rajneesh Kumar², Anita Goel ${ }^{1}$, and Aseem Miglani ${ }^{1}$ \\ ${ }^{1}$ Department of Mathematics, Maharshi Dayanand University, Rohtak-124001, India \\ ${ }^{2}$ Department of Mathematics, Kurukshetra University, Kurukshetra-136 119, India
}

(Received February 18, 2002; Revised August 30, 2002; Accepted September 6, 2002)

\begin{abstract}
The analytic expressions for the displacements and stresses at any point of an infinite orthotropic elastic medium as a result of an inclined line load have been obtained. This plane strain problem has been solved by using eigenvalue approach and the use of matrix notation avoids unwieldy mathematical expressions. The technique developed in the present paper is simple, straightforward and convenient for numerical computation. The variations of the displacements and stresses with the horizontal distance have been shown graphically.
\end{abstract}

\section{Introduction}

The slightest departure from isotropy is transversely isotropy in which there is one axis of symmetry and there are five elastic constants instead of two in an isotropic material. Crystals of close-packed hexagonal form belong to the class of such materials. A medium with three mutually orthogonal planes of symmetry is known as orthorhombic. When one of the plane of symmetry in an orthorhombic symmetry is horizontal, the symmetry is termed as orthotropic symmetry (Crampin, 1989). The orthotropy symmetry is exhibited by olivine and orthopyroxenes, the principal rock-forming minerals of deep crust and upper mantle. Dziewonski and Anderson (1981) have established that the upper part of the earth is anisotropic.

When the source surface is very long in one direction in comparison with the others, the use of two-dimensional approximation is justified and consequently calculations are simplified to a great extent and one gets a closed form analytical solution. A very long strip-source and a very long linesource are examples of such two-dimensional sources. Love (1944) obtained expressions for the displacements due to a line source in an isotropic elastic medium. Maruyama (1966) obtained the displacement and stress fields corresponding to long strike-slip faults in a homogeneous isotropic half-space. Okada $(1985,1992)$ provided a compact analytic expressions for the surface deformation and internal deformation due to inclined shear and tensile faults in a homogeneous isotropic half space.

Using the body-force equivalent of dislocation source as discussed by Burridge and Knopoff (1964) and Aki and Richards (1980), Pan (1989) obtained the response of a transversely isotropic layered medium to general dislocation sources. Garg et al. (1996) obtained the representation of seismic sources causing antiplane strain deformation of an

Copy right(C) The Society of Geomagnetism and Earth, Planetary and Space Sciences (SGEPSS); The Seismological Society of Japan; The Volcanological Society of Japan; The Geodetic Society of Japan; The Japanese Society for Planetary Sciences. orthotropic medium. Kumar et al. (2000) used eigenvalue approach to solve the plane strain problem of poroelasticity for an isotropic medium. The corresponding problem for a transversely isotropic medium has been discussed by Kumar et al. (2002).

Considering the earth model as orthotropic instead of isotropic for better approximation, in the present paper, we study the general plane-strain problem of an infinite orthotropic elastic-medium due to two-dimensional sources. The eigenvalue approach has been used to solve it. We have obtained the closed-form expressions for the twodimensional displacements and stresses at any point of an infinite orthotropic medium due to an inclined line load. The deformation due to other two-dimensional sources such as strip loads, continuous line loads, etc., can also be similarly obtained. The deformation at any point of the medium is useful to analyse the deformation field around mining tremors and drilling into the crust of the earth. It can also contribute to the theoretical consideration of the seismic and volcanic sources since it can account for the deformation fields in the entire volume surrounding the source region. It may also find application in various engineering problems regarding the deformation of an orthotropic medium. One may also find the deformation due to seismic sources by differentiation and addition.

\section{Basic Equations}

The equilibrium equations in the Cartesian co-ordinate system $\left(x_{1}, x_{2}, x_{3}\right)$ for zero body forces are

$$
\begin{aligned}
& \frac{\partial \tau_{11}}{\partial x_{1}}+\frac{\partial \tau_{12}}{\partial x_{2}}+\frac{\partial \tau_{13}}{\partial x_{3}}=0, \\
& \frac{\partial \tau_{21}}{\partial x_{1}}+\frac{\partial \tau_{22}}{\partial x_{2}}+\frac{\partial \tau_{23}}{\partial x_{3}}=0, \\
& \frac{\partial \tau_{31}}{\partial x_{1}}+\frac{\partial \tau_{32}}{\partial x_{2}}+\frac{\partial \tau_{33}}{\partial x_{3}}=0,
\end{aligned}
$$

where $\tau_{i j}(i, j=1,2,3)$ are the components of stress tensor. 
The stress-strain relations for an orthotropic elastic medium, with co-ordinate planes as planes, of elastic symmetry, are (Mase and Mase, 1999)

$$
\begin{aligned}
& \tau_{11}=d_{11} e_{11}+d_{12} e_{22}+d_{13} e_{33}, \\
& \tau_{22}=d_{12} e_{11}+d_{22} e_{22}+d_{23} e_{33}, \\
& \tau_{33}=d_{13} e_{11}+d_{23} e_{22}+d_{33} e_{33}, \\
& \tau_{23}=2 d_{44} e_{23}, \\
& \tau_{13}=2 d_{55} e_{13}, \\
& \tau_{12}=2 d_{66} e_{12},
\end{aligned}
$$

where $e_{i j}(i, j=1,2,3)$ are the components of the strain tensor and are related with displacement components $\left(u_{1}, u_{2}, u_{3}\right)$ through the relations

$$
e_{i j}=\frac{1}{2}\left(\frac{\partial u_{i}}{\partial x_{j}}+\frac{\partial u_{j}}{\partial x_{i}}\right), \quad 1 \leq i, j \leq 3 .
$$

The two-suffix quantity $d_{i j}(i, j=1,2, \ldots, 6)$ are the elastic moduli for the orthotropic elastic medium.

For convenience, we shall write $\left(x_{1}, x_{2}, x_{3}\right)=(x, y, z)$ and $\left(u_{1}, u_{2}, u_{3}\right)=(u, v, w)$.

The equilibrium equations in terms of displacement components can be obtained from Eqs. (1)-(5). We find

$$
\begin{aligned}
& d_{11} \frac{\partial^{2} u}{\partial x^{2}}+d_{66} \frac{\partial^{2} u}{\partial y^{2}}+d_{55} \frac{\partial^{2} u}{\partial z^{2}}+\left(d_{12}+d_{66}\right) \frac{\partial^{2} v}{\partial x \partial y} \\
& +\left(d_{13}+d_{55}\right) \frac{\partial^{2} w}{\partial x \partial z}=0, \\
& \left(d_{66}+d_{12}\right) \frac{\partial^{2} u}{\partial x \partial y}+d_{66} \frac{\partial^{2} v}{\partial x^{2}}+d_{22} \frac{\partial^{2} v}{\partial y^{2}}+d_{44} \frac{\partial^{2} v}{\partial z^{2}} \\
& +\left(d_{23}+d_{44}\right) \frac{\partial^{2} w}{\partial z \partial y}=0, \\
& \left(d_{55}+d_{13}\right) \frac{\partial^{2} u}{\partial x \partial z}+\left(d_{44}+d_{23}\right) \frac{\partial^{2} v}{\partial z \partial y}+d_{55} \frac{\partial^{2} w}{\partial x^{2}} \\
& +d_{44} \frac{\partial^{2} w}{\partial y^{2}}+d_{33} \frac{\partial^{2} w}{\partial z^{2}}=0 .
\end{aligned}
$$

\section{Formulation of the Problem}

We consider an infinite orthotropic elastic medium with $x$-axis vertically downwards. Suppose that an inclined line load $F_{0}$, per unit length, is acting on the $z$-axis and its inclination with $x$-direction is $\delta$ (Fig. 1). This is a twodimensional plane strain problem. We shall calculate the resulting stresses and displacements at any point of an orthotropic infinite elastic medium.

\section{Theory}

We consider plane strain deformation, parallel to $x y$ plane, in which the displacement components are independent of $z$ and are of the type

$$
u=u(x, y), \quad v=v(x, y), \quad w \equiv 0 .
$$

The non-zero stresses for the plane strain problem are

$$
\tau_{11}=d_{11} \frac{\partial u}{\partial x}+d_{12} \frac{\partial v}{\partial y}
$$

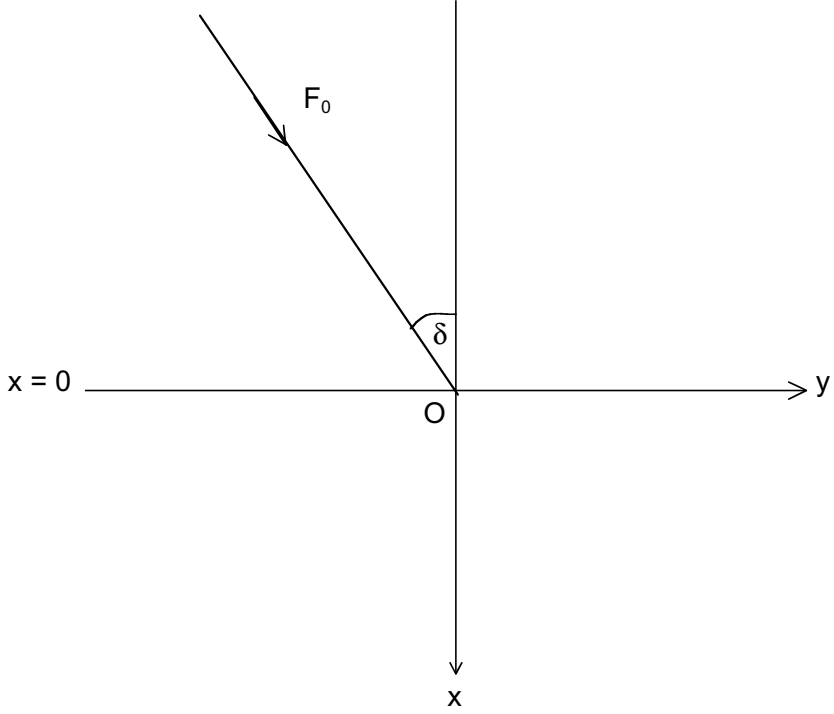

Fig. 1. $z=0$ section of the infinite orthotropic medium.

$$
\begin{aligned}
\tau_{22} & =d_{12} \frac{\partial u}{\partial x}+d_{22} \frac{\partial v}{\partial y}, \\
\tau_{33} & =d_{13} \frac{\partial u}{\partial x}+d_{23} \frac{\partial v}{\partial y}, \\
\tau_{12} & =d_{66}\left(\frac{\partial u}{\partial y}+\frac{\partial v}{\partial x}\right) .
\end{aligned}
$$

The equilibrium equations for an orthotropic elastic medium due to plane strain deformation are to be found from Eqs. (6)-(9). We find

$$
\begin{aligned}
& d_{11} \frac{\partial^{2} u}{\partial x^{2}}+d_{66} \frac{\partial^{2} u}{\partial y^{2}}+\left(d_{66}+d_{12}\right) \frac{\partial^{2} v}{\partial x \partial y}=0 \\
& \left(d_{66}+d_{12}\right) \frac{\partial^{2} u}{\partial x \partial y}+d_{66} \frac{\partial^{2} v}{\partial x^{2}}+d_{22} \frac{\partial^{2} v}{\partial y^{2}}=0
\end{aligned}
$$

and equilibrium equation (8) becomes an identity.

We define Fourier transform $\bar{f}(x, k)$ of $f(x, y)$ by the relation (Debnath, 1995)

$$
\bar{f}(x, k)=F[f(x, y)]=\int_{-\infty}^{\infty} f(x, y) e^{i k y} d y
$$

so that

$$
f(x, y)=\frac{1}{2 \pi} \int_{-\infty}^{\infty} \bar{f}(x, k) e^{-i k y} d k,
$$

where $k$ is the transformed Fourier parameter. We know that

$$
F\left(\frac{\partial}{\partial y} f(x, y)\right)=(-i k) \bar{f}(x, k),
$$

and

$$
F\left(\frac{\partial^{2}}{\partial y^{2}} f(x, y)\right)=-k^{2} \bar{f}(x, k) .
$$

Applying the Fourier transformation on equilibrium equations (14) and (15) for an orthotropic elastic medium, we 
find

$$
\begin{aligned}
& d_{11} \frac{d^{2} \bar{u}}{d x^{2}}-k^{2} d_{66} \bar{u}+\left(d_{66}+d_{12}\right)(-i k) \frac{d \bar{v}}{d x}=0, \\
& \left(d_{66}+d_{12}\right)(-i k) \frac{d \bar{u}}{d x}+d_{66} \frac{d^{2} \bar{v}}{d x^{2}}+d_{22}\left(-k^{2} \bar{v}\right)=0 .
\end{aligned}
$$

The above two equations can be written in the following vector-matrix differential equation

$$
A \frac{d^{2} W}{d x^{2}}-i k B \frac{d W}{d x}-k^{2} C W=0,
$$

where

$$
\begin{array}{ll}
A=\left(\begin{array}{cc}
d_{11} & 0 \\
0 & d_{66}
\end{array}\right), & B=\left(\begin{array}{cc}
0 & d_{66}+d_{12} \\
d_{66}+d_{12} & 0
\end{array}\right), \\
C=\left(\begin{array}{cc}
d_{66} & 0 \\
0 & d_{22}
\end{array}\right), & W=\left(\begin{array}{l}
\bar{u} \\
\bar{v}
\end{array}\right) .
\end{array} .
$$

We note that matrices $A, B, C$ are all symmetric. Now we try a solution of the matrix equation (22) of the form

$$
W(x, k)=E(k) e^{m x},
$$

where $m$ is a parameter and $E(k)$ is a matrix of the type $2 \times 1$.

Substitution of the value of $W$ from Eq. (24) into Eq. (22) gives the following characteristic equation.

$$
\begin{aligned}
& \left(d_{11} d_{66}\right) m^{4}-\left(d_{11} d_{22}-2 d_{66} d_{12}-d_{12}^{2}\right) k^{2} m^{2} \\
& +\left(d_{66} d_{22}\right) k^{4}=0 .
\end{aligned}
$$

A transversely isotropy, with $z$-axis coinciding with the axis of symmetry, is a particular case of orthotropy in which (Payton, 1983)

$$
\begin{array}{ll}
d_{22}=d_{11}, & d_{23}=d_{13}, \\
d_{55}=d_{44}, & d_{66}=1 / 2\left(d_{11}-d_{12}\right) .
\end{array}
$$

The characteristic equation (25) for a transversely isotropic medium becomes

$$
m^{4}-2 k^{2} m^{2}+k^{4}=0,
$$

provided $d_{11} \neq 0$ and $d_{11} \neq d_{12}$. We observe that the characteristic equation (27) for a transversely isotropic medium is independent of its elastic moduli.

When the medium is isotropic (Payton, 1983), then

$$
\begin{aligned}
& d_{11}=d_{22}=d_{33}=\lambda+2 \mu, \quad d_{12}=d_{13}=d_{23}=\lambda, \\
& d_{44}=d_{55}=d_{66}=\mu .
\end{aligned}
$$

where $\lambda$ and $\mu$ are the Lamé elastic moduli. The characteristic equation (25) for an isotropic elastic medium becomes

$$
m^{4}-2 k^{2} m^{2}+k^{4}=0,
$$

which is independent of $\lambda$ and $\mu$. From above, we notice that the characteristic equation is the same for both types of elastic symmetries - transversely and isotropic.

The solution of characteristic equation (25) gives the eigenvalues, in case of an orthotropic elastic medium, as

$$
m^{2}=\alpha_{1}^{2} k^{2}, \alpha_{2}^{2} k^{2}
$$

where

$$
\begin{aligned}
& \alpha_{1}^{2}=\frac{A_{0}+\sqrt{A_{0}^{2}-4 B_{0}}}{2}, \quad \alpha_{2}^{2}=\frac{A_{0}-\sqrt{A_{0}^{2}-4 B_{0}}}{2}, \\
& A_{0}=\frac{d_{11} d_{22}-2 d_{66} d_{12}-d_{12}^{2}}{d_{11} d_{66}}, \quad B_{0}=d_{22} / d_{11} .
\end{aligned}
$$

We note that $\alpha_{1}$ and $\alpha_{2}$ for an orthotropic elastic medium depends upon elastic moduli only (and not on $k$ ) and they may be real or complex. We assume that $\alpha_{1} \neq \alpha_{2}$ for an orthotropic medium. Then, the eigenvalues may be written as

$$
\begin{aligned}
& m_{1}=\alpha_{1}|k|, \quad m_{2}=\alpha_{2}|k|, \\
& m_{3}=-\alpha_{1}|k|, \quad m_{4}=-\alpha_{2}|k|,
\end{aligned}
$$

with real parts of $\left\{\alpha_{1}, \alpha_{2}\right\}$ as positive.

The eigenvalues for the orthotropic elastic medium are obtained by solving the matrix equation

$$
\left[m^{2} A-i m k B-k^{2} C\right] E(k)=0,
$$

in which the matrices $A, B, C$ are given by Eq. (23). The eigenvectors are found to be

$$
E_{N}^{T}=\left[P_{N}, 1\right], \quad E_{N+2}^{T}=\left[-P_{N}, 1\right],
$$

where

$$
\begin{aligned}
P_{N} & =i \frac{\alpha_{N}\left(d_{66}+d_{12}\right)}{\alpha_{N}^{2} d_{11}-d_{66}} \\
& =-i \frac{\alpha_{N}^{2} d_{66}-d_{22}}{\alpha_{N}\left(d_{66}+d_{12}\right)} \quad \text { for } \quad N=1,2 .
\end{aligned}
$$

For a transversely isotropic medium as well as for a perfectly isotropic elastic medium, we find that

$$
\alpha_{N}=1, \quad P_{N}=i \quad \text { for } \quad N=1,2 .
$$

Thus, a solution of matrix equation (22) for the case of an orthotropic elastic medium is

$$
W(x, k)=\sum_{N=1}^{2}\left(B_{N} E_{N}^{T} e^{\alpha_{N}|k| x}+B_{N+2} E_{N+2}^{T} e^{-\alpha_{N}|k| x}\right) .
$$

Where $B_{1}, B_{2}, B_{3}, B_{4}$ are constants to be determined from boundary conditions and they may depend upon $k$. From Eqs. (23), (35) and (38), we write

$$
\begin{aligned}
\bar{u}(x, k)= & B_{1} P_{1} e^{\alpha_{1}|k| x}+B_{2} P_{2} e^{\alpha_{2}|k| x}-B_{3} P_{1} e^{-\alpha_{1}|k| x} \\
& -B_{4} P_{2} e^{-\alpha_{2}|k| x} \\
\bar{v}(x, k)= & B_{1} e^{\alpha_{1}|k| x}+B_{2} e^{\alpha_{2}|k| x}+B_{3} e^{-\alpha_{1}|k| x} \\
& +B_{4} e^{-\alpha_{2}|k| x}
\end{aligned}
$$

Inversion of Eqs. (39) and (40) yield the displacements in the following integral forms

$$
\begin{aligned}
u(x, y)= & \frac{1}{2 \pi} \int_{-\infty}^{\infty}\left(B_{1} P_{1} e^{\alpha_{1}|k| x}+B_{2} P_{2} e^{\alpha_{2}|k| x}\right. \\
& \left.-B_{3} P_{1} e^{-\alpha_{1}|k| x}-B_{4} P_{2} e^{-\alpha_{2}|k| x}\right) e^{-i k y} d k, \\
v(x, y)= & \frac{1}{2 \pi} \int_{-\infty}^{\infty}\left(B_{1} e^{\alpha_{1}|k| x}+B_{2} e^{\alpha_{2}|k| x}\right. \\
& \left.+B_{3} e^{-\alpha_{1}|k| x}+B_{4} e^{-\alpha_{2}|k| x}\right) e^{-i k y} d k
\end{aligned}
$$


The stress components in integral forms for an orthotropic elastic medium due to plane strain deformation are obtained from Eqs. (14), (41) and (42). We find

$$
\begin{aligned}
\tau_{11}= & \frac{1}{2 \pi} \int_{-\infty}^{\infty}\left(Q_{1} B_{1} e^{\alpha_{1}|k| x}+Q_{2} B_{2} e^{\alpha_{2}|k| x}\right. \\
& \left.+Q_{1} B_{3} e^{-\alpha_{1}|k| x}+Q_{2} B_{4} e^{-\alpha_{2}|k| x}\right) e^{-i k y} d k, \\
\tau_{12}= & \frac{1}{2 \pi} \int_{-\infty}^{\infty} d_{66}\left(R_{1} B_{1} e^{\alpha_{1}|k| x}+R_{2} B_{2} e^{\alpha_{2}|k| x}\right. \\
& \left.-R_{1} B_{3} e^{-\alpha_{1}|k| x}-R_{2} B_{4} e^{-\alpha_{2}|k| x}\right) e^{-i k y} d k,
\end{aligned}
$$

where

$$
\begin{aligned}
& Q_{N}=d_{11} P_{N} \alpha_{N}|k|-i d_{12} k, \\
& R_{N}=\alpha_{N}|k|-i P_{N} k, \quad N=1,2 .
\end{aligned}
$$

\section{Solution of the Problem}

To find the displacements and stresses at any point of an orthotropic infinite elastic medium due to an inclined line load $F_{0}$, per unit length, acting on the $z$-axis, we consider the infinite medium as consisting of Medium $\mathrm{I}(x>0)$ and Medium II $(x<0)$ of identical elastic properties.

The displacement and stress components for the Medium I are

$$
\begin{aligned}
u^{\mathrm{I}}(x, y)= & \frac{1}{2 \pi} \int_{-\infty}^{\infty}\left(-B_{3} P_{1} e^{-\alpha_{1}|k| x}\right. \\
& \left.-B_{4} P_{2} e^{-\alpha_{2}|k| x}\right) e^{-i k y} d k \\
v^{\mathrm{I}}(x, y)= & \frac{1}{2 \pi} \int_{-\infty}^{\infty}\left(B_{3} e^{-\alpha_{1}|k| x}+B_{4} e^{-\alpha_{2}|k| x}\right) e^{-i k y} d k \\
\tau_{11}^{\mathrm{I}}(x, y)= & \frac{1}{2 \pi} \int_{-\infty}^{\infty}\left(Q_{1} B_{3} e^{-\alpha_{1}|k| x}\right. \\
& \left.+Q_{2} B_{4} e^{-\alpha_{2}|k| x}\right) e^{-i k y} d k \\
\tau_{12}^{\mathrm{I}}(x, y)= & \frac{1}{2 \pi} \int_{-\infty}^{\infty} d_{66}\left(-R_{1} B_{3} e^{-\alpha_{1}|k| x}\right. \\
& \left.-R_{2} B_{4} e^{-\alpha_{2}|k| x}\right) e^{-i k y} d k
\end{aligned}
$$

and the displacement and stress components for the Medium II are

$$
\begin{aligned}
u^{\mathrm{II}}(x, y)= & \frac{1}{2 \pi} \int_{-\infty}^{\infty}\left(B_{1} P_{1} e^{\alpha_{1}|k| x}\right. \\
& \left.+B_{2} P_{2} e^{\alpha_{2}|k| x}\right) e^{-i k y} d k \\
v^{\mathrm{II}}(x, y)= & \frac{1}{2 \pi} \int_{-\infty}^{\infty}\left(B_{1} e^{\alpha_{1}|k| x}+B_{2} e^{\alpha_{2}|k| x}\right) e^{-i k y} d k, \\
\tau_{11}^{\mathrm{II}}(x, y)= & \frac{1}{2 \pi} \int_{-\infty}^{\infty}\left(Q_{1} B_{1} e^{\alpha_{1}|k| x}\right. \\
& \left.+Q_{2} B_{2} e^{\alpha_{2}|k| x}\right) e^{-i k y} d k, \\
\tau_{12}^{\mathrm{II}}(x, y)= & \frac{1}{2 \pi} \int_{-\infty}^{\infty} d_{66}\left(R_{1} B_{1} e^{\alpha_{1}|k| x}\right. \\
& \left.+R_{2} B_{2} e^{\alpha_{2}|k| x}\right) e^{-i k y} d k .
\end{aligned}
$$

\subsection{Normal line load}

Consider a normal line load $F_{1}$, per unit length, acting in the positive $x$-direction on the interface $x=0$ along the $z$ -

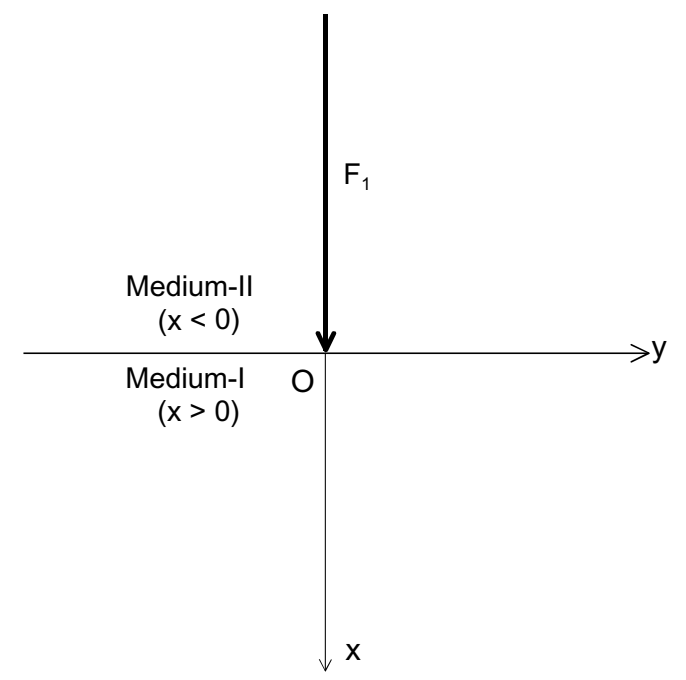

Fig. 2. A normal line load.

axis (Fig. 2). Then the boundary conditions at $x=0$ are

$$
\begin{aligned}
& u^{\mathrm{I}}(x, y)-u^{\mathrm{II}}(x, y)=0, \quad v^{\mathrm{I}}(x, y)-v^{\mathrm{II}}(x, y)=0, \\
& \tau_{11}^{\mathrm{I}}(x, y)-\tau_{11}^{\mathrm{II}}(x, y)=-F_{1} \delta(y) \\
& \tau_{12}^{\mathrm{I}}(x, y)-\tau_{12}^{\mathrm{II}}(x, y)=0
\end{aligned}
$$

where $\delta(y)$ is the Dirac delta function satisfying the following properties

$$
\int_{-\infty}^{\infty} \delta(y) d y=1, \quad \delta(y)=\frac{1}{2 \pi} \int_{-\infty}^{\infty} e^{-i k y} d k .
$$

From Eqs. (46) to (56), we find the values of coefficients for a normal line load as below.

$$
B_{4}=-B_{3}=-B_{2}=B_{1}=\frac{-F_{1}}{2\left(Q_{2}-Q_{1}\right)},
$$

where

$$
Q_{2}-Q_{1}=d_{11}\left(P_{2} \alpha_{2}-P_{1} \alpha_{1}\right)|k| \text {. }
$$

Substituting the values of various constants $B_{i}$ 's from Eqs. (57) and (58) into Eqs. (46) to (53) and using the standard integrals (see, Appendix), we find the following closed-form expressions for the displacements and stresses at any point of an orthotropic infinite elastic medium as a result of a normal line load.

$$
\begin{aligned}
& u^{N}(x, y)=\frac{F_{1}}{4 \pi d_{11}\left(P_{2} \alpha_{2}-P_{1} \alpha_{1}\right)} \\
& \times\left(P_{1} \log \left(y^{2}+\alpha_{1}^{2} x^{2}\right)-P_{2} \log \left(y^{2}+\alpha_{2}^{2} x^{2}\right)\right), \\
& v^{N}(x, y)=\mp \frac{F_{1}}{4 \pi d_{11}\left(P_{2} \alpha_{2}-P_{1} \alpha_{1}\right)} \\
& \times\left(\log \left(y^{2}+\alpha_{1}^{2} x^{2}\right)-\log \left(y^{2}+\alpha_{2}^{2} x^{2}\right)\right), \\
& \tau_{11}^{N}(x, y)=\frac{x F_{1}}{2 \pi\left(P_{2} \alpha_{2}-P_{1} \alpha_{1}\right)} \\
& \times\left(\frac{y^{2}\left(P_{1} \alpha_{1}^{2}-P_{2} \alpha_{2}^{2}\right)+\alpha_{1}^{2} \alpha_{2}^{2} x^{2}\left(P_{1}-P_{2}\right)}{\left(y^{2}+\alpha_{1}^{2} x^{2}\right)\left(y^{2}+\alpha_{2}^{2} x^{2}\right)}\right)
\end{aligned}
$$




$$
\begin{aligned}
& \pm \frac{d_{12}\left(\alpha_{1}^{2}-\alpha_{2}^{2}\right) x^{2} y F_{1}}{2 \pi d_{11}\left(P_{2} \alpha_{2}-P_{1} \alpha_{1}\right)\left(y^{2}+\alpha_{1}^{2} x^{2}\right)\left(y^{2}+\alpha_{2}^{2} x^{2}\right)} \\
& \tau_{12}^{N}(x, y)=\frac{y d_{66} F_{1}}{2 \pi d_{11}\left(P_{2} \alpha_{2}-P_{1} \alpha_{1}\right)} \\
& \times\left(\frac{y^{2}\left(P_{1}-P_{2}\right) \mp x y\left(\alpha_{1}^{2}-\alpha_{2}^{2}\right)+x^{2}\left(P_{1} \alpha_{2}^{2}-P_{2} \alpha_{1}^{2}\right)}{\left(y^{2}+\alpha_{1}^{2} x^{2}\right)\left(y^{2}+\alpha_{2}^{2} x^{2}\right)}\right)
\end{aligned}
$$

where the upper sign is for Medium I and the lower sign for Medium II and superscript $(N)$ indicates the deformation due to a normal line load $F_{1}$.

\subsection{Tangential line load}

Assume that a line force $F_{2}$, per unit length, is acting at the origin in the positive $y$-direction (Fig. 3 ). Then, the boundary conditions at the horizontal plane $x=0$ are

$$
\begin{aligned}
& u^{\mathrm{I}}(x, y)-u^{\mathrm{II}}(x, y)=0, \quad v^{\mathrm{I}}(x, y)-v^{\mathrm{II}}(x, y)=0, \\
& \tau_{11}^{\mathrm{I}}(x, y)-\tau_{11}^{\mathrm{II}}(x, y)=0, \\
& \tau_{12}^{\mathrm{I}}(x, y)-\tau_{12}^{\mathrm{II}}(x, y)=-F_{2} \delta(y),
\end{aligned}
$$

where $\delta(y)$ is the Dirac delta function. From Eqs. (46) to (53) and using the boundary conditions given in Eqs. (63) and (64), we find the following values of the coefficients $B_{i}$ for a tangential line load.

$$
\begin{aligned}
& B_{1}=B_{3}=\frac{F_{2} P_{2}}{2 d_{66}\left(P_{2} R_{1}-P_{1} R_{2}\right)}, \\
& B_{2}=B_{4}=\frac{-F_{2} P_{1}}{2 d_{66}\left(P_{2} R_{1}-P_{1} R_{2}\right)},
\end{aligned}
$$

where

$$
P_{2} R_{1}-P_{1} R_{2}=\left(P_{2} \alpha_{1}-P_{1} \alpha_{2}\right)|k| .
$$

Substituting the values of various constants $B_{i}$ 's from Eqs. (65) to (67) into Eqs. (46) to (53) and then using the standard integrals (see, Appendix), we find the following closed-form expressions for the displacements and stresses at any point of an orthotropic infinite elastic medium as a result of tangential line load, $F_{2}$.

$$
\begin{aligned}
& u^{T}(x, y)= \pm \frac{F_{2} P_{1} P_{2}}{4 \pi d_{66}\left(P_{2} \alpha_{1}-P_{1} \alpha_{2}\right)} \\
& \times\left[\log \left(y^{2}+\alpha_{1}^{2} x^{2}\right)-\log \left(y^{2}+\alpha_{2}^{2} x^{2}\right)\right],
\end{aligned}
$$

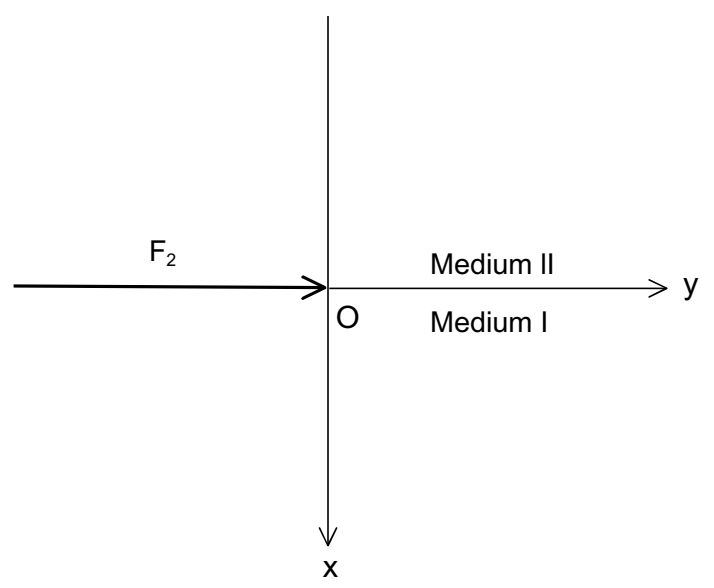

Fig. 3. A tangential line load.

$$
\begin{aligned}
& v^{T}(x, y)=\frac{-F_{2}}{4 \pi d_{66}\left(P_{2} \alpha_{1}-P_{1} \alpha_{2}\right)} \\
& \times\left[P_{2} \log \left(y^{2}+\alpha_{1}^{2} x^{2}\right)-P_{1} \log \left(y^{2}+\alpha_{2}^{2} x^{2}\right)\right], \\
& \tau_{11}^{T}(x, y)=\frac{d_{12} y F_{2}}{2 \pi d_{66}\left(P_{2} \alpha_{1}-P_{1} \alpha_{2}\right)} \\
& \times\left(\frac{y^{2}\left(P_{1}-P_{2}\right)+x^{2}\left(P_{1} \alpha_{1}^{2}-P_{2} \alpha_{2}^{2}\right)}{\left(y^{2}+\alpha_{1}^{2} x^{2}\right)\left(y^{2}+\alpha_{2}^{2} x^{2}\right)}\right) \\
& \pm \frac{d_{11} P_{1} P_{2}\left(\alpha_{1}^{2}-\alpha_{2}^{2}\right) x y^{2} F_{2}}{2 \pi d_{66}\left(P_{2} \alpha_{1}-P_{1} \alpha_{2}\right)\left(y^{2}+\alpha_{1}^{2} x^{2}\right)\left(y^{2}+\alpha_{2}^{2} x^{2}\right)} \\
& \tau_{12}^{T}(x, y)=\frac{x F_{2}}{2 \pi\left(P_{2} \alpha_{1}-P_{1} \alpha_{2}\right)} \\
& \times\left(\frac{\alpha_{1}^{2} \alpha_{2}^{2} x^{2}\left(P_{1}-P_{2}\right)+y^{2}\left(P_{1} \alpha_{2}^{2}-P_{2} \alpha_{1}^{2}\right)}{\left(y^{2}+\alpha_{1}^{2} x^{2}\right)\left(y^{2}+\alpha_{2}^{2} x^{2}\right)}\right) \\
& +\frac{P_{1} P_{2}\left(\alpha_{1}^{2}-\alpha_{2}^{2}\right) x^{2} y F_{2}}{\left(y^{2}+\alpha_{1}^{2} x^{2}\right)\left(y^{2}+\alpha_{2}^{2} x^{2}\right)}
\end{aligned}
$$

where the upper sign is for Medium I and the lower sign for Medium II and $(T)$ indicate results due to a tangential line load.

\subsection{Inclined line load}

For an inclined line load $F_{0}$, per unit length, we have (see, Fig. 1) (Saada, 1974)

$$
F_{1}=F_{0} \cos \delta, \quad F_{2}=F_{0} \sin \delta .
$$

The stresses and displacements subjected to inclined load can be obtained by superposition of the vertical and tangential cases. The final deformation of the formulated problem is given by

$$
\begin{aligned}
& u^{(I N)}(x, y)=u^{(N)}(x, y)+u^{(T)}(x, y), \\
& v^{(I N)}(x, y)=v^{(N)}(x, y)+v^{(T)}(x, y), \\
& \tau_{12}^{(I N)}(x, y)=\tau_{12}^{(N)}(x, y)+\tau_{12}^{(T)}(x, y), \\
& \tau_{11}^{(I N)}(x, y)=\tau_{11}^{(N)}(x, y)+\tau_{11}^{(T)}(x, y),
\end{aligned}
$$

where deformations due to a normal line load $F_{1}$ and a tangential line load $F_{2}$ are obtained earlier. The superscript $(I N)$ indicates results due to an inclined line load $F_{0}$.

\section{Numerical Results}

In Eqs. (73) to (76) we have obtained the closed form expressions for the displacement and stress components at any point of an infinite orthotropic elastic medium as a result of an inclined line load $F_{0}$, per unit length, acting on the $z$ axis with its inclination $\delta$ with the $x$-direction. For numerical computation, we use the values of elastic constants given by Love (1944) for Topaz material, which are

$$
\begin{aligned}
& d_{11}=2870, \quad d_{22}=3560, \quad d_{33}=3000, \\
& d_{12}=1280, \quad d_{23}=900, \quad d_{13}=860, \\
& d_{44}=1100, \quad d_{55}=1350, \quad d_{66}=1330,
\end{aligned}
$$

in terms of a unit stress of $10^{6}$ grammes wt. $/ \mathrm{cm}^{2}$.

We have plotted graphs in Figs. 4-7 for the variation of displacements and stresses against the horizontal distance $y$ for a fixed value of $x=1.0$. Each figure has four curves corresponding to four different values of $\delta$, namely, $\delta=0^{\circ}$, 


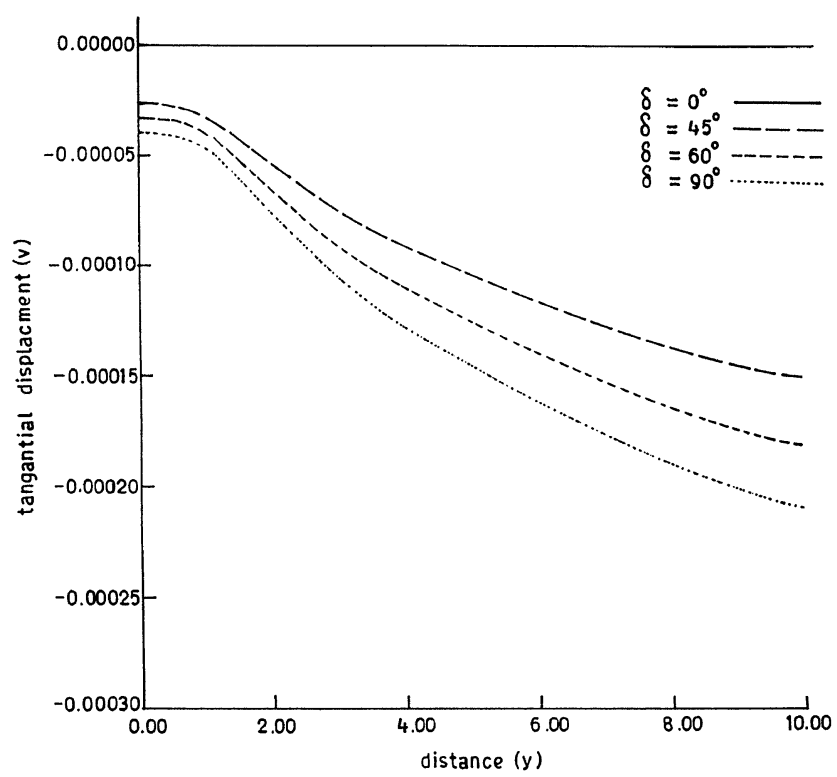

Fig. 4. Variation of tangential displacement $(v)$ against the horizontal distance $(y)$ on the plane $x=1.0$.

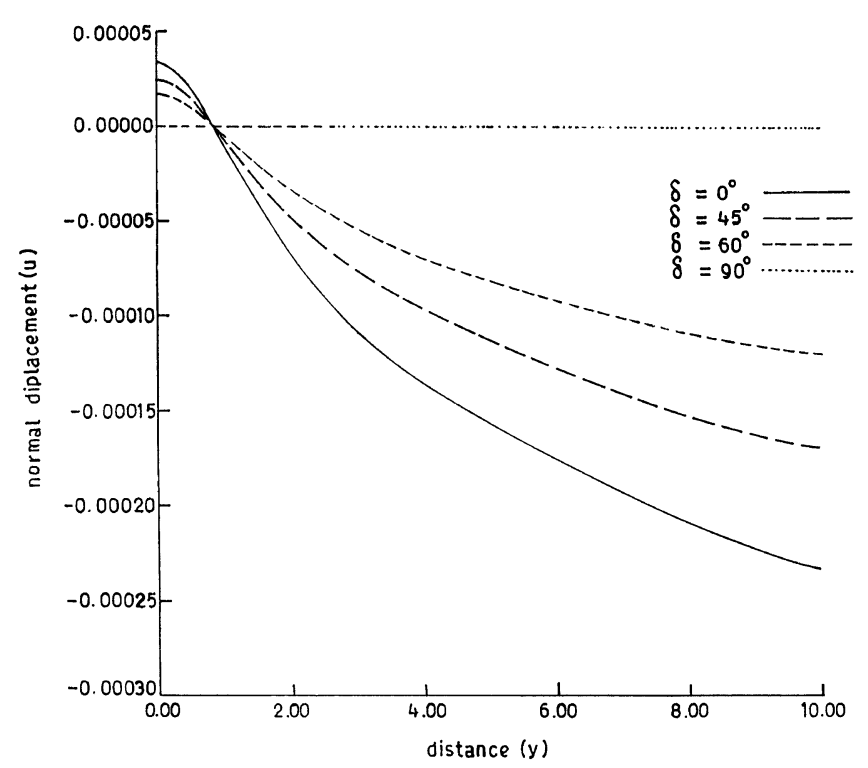

Fig. 5. Variation of normal displacement $(u)$ against the horizontal distance (y) on the plane $x=1.0$

$45^{\circ}, 60^{\circ}$ and $90^{\circ}$. The case $\delta=0^{\circ}$ corresponds to a normal line load and $\delta=90^{\circ}$ for a tangential line load.

Figures 4-5 exhibit the graphs for the variation of tangential displacement $(V)$ and the variation of normal displacement $(U)$, respectively. These figures show that the displacements for $\delta=45^{\circ}, 60^{\circ}$ lie between the corresponding displacements for a normal line load and tangential line load.

Figures 6-7 correspond to the variation of tangential and normal stresses. In Fig. 6, the curves for different values of $\delta$ change steadily. Figure 7 points frequent inter-crossing of various curves for different values of $\delta$.

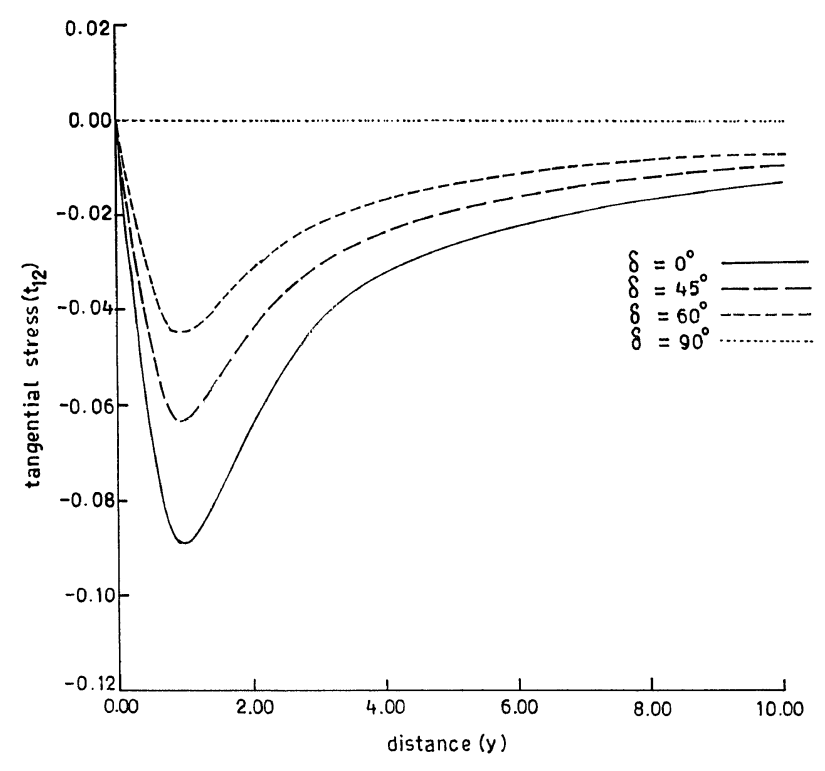

Fig. 6. Variation of tangential stress $\left(t_{12}\right)$ against the horizontal distance (y) on the plane $x=1.0$.

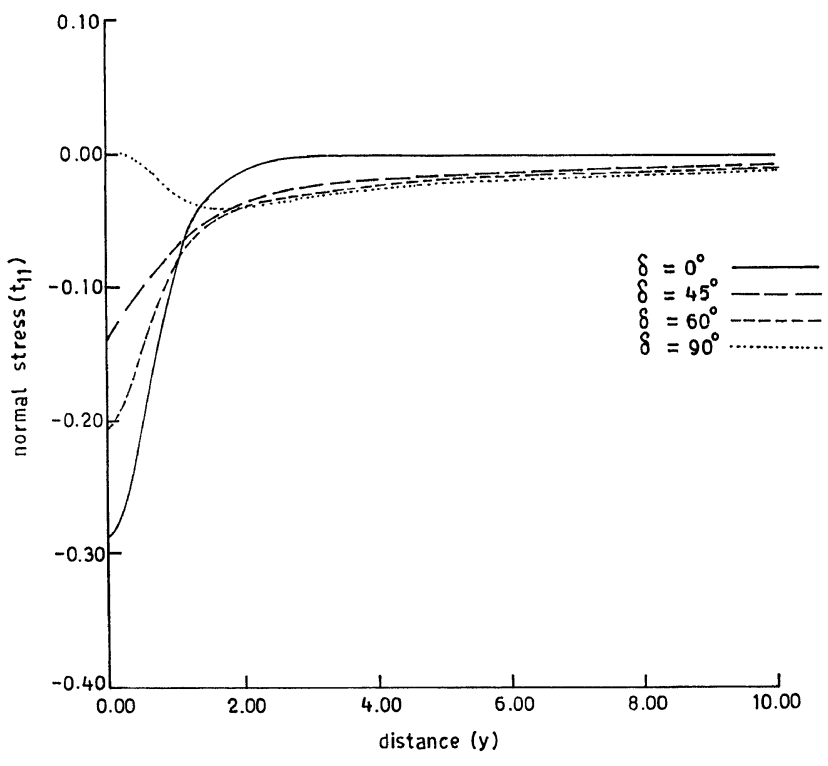

Fig. 7. Variation of normal stress $\left(t_{11}\right)$ against the horizontal distance $(y)$ on the plane $x=1.0$.

Acknowledgments. The authors are extremely grateful to the referee, Dr. Yoshimitsu Okada for giving valuable suggestions which lead the paper in its present form.

Appendix A. $(\xi>0)$

$$
\begin{aligned}
& \int_{-\infty}^{\infty} \exp (-|k| \xi) \exp (-i k y) d k=\frac{2 \xi}{y^{2}+\xi^{2}} \\
& \int_{-\infty}^{\infty} \exp (-|k| \xi) \frac{k}{|k|} \exp (-i k y) d k=\frac{-2 i y}{y^{2}+\xi^{2}} \\
& \int_{-\infty}^{\infty}(|k|)^{-1} \exp (-|k| \xi) \exp (-i k y) d k=-\log \left(y^{2}+\xi^{2}\right)
\end{aligned}
$$




\section{References}

Aki, K. and P. G. Richards, Quantitative Seismology. Theory and Methods, W. H. Freeman, San Francisco, 932, 1980.

Burridge, R. and L. Knopoff, Body force equivalents for seismic dislocations, Bull. Seismol. Soc. Am., 54, 1875-1888, 1964.

Crampin, S., Suggestions for a consistent terminology for seismic anisotropy, Geophys. Prospect., 37, 753-770, 1989.

Debnath, L., Integral Transforms and their Application, CRC Press. Inc., New York, 1995.

Dziewonski, A. M. and D. L. Anderson, Preliminary reference earth model, Phys. Earth Planet. Inter, 25, 297-356, 1981.

Garg, N. R., D. K. Madan, and R. K. Sharma, Two-dimensional deformation of an orthotropic elastic medium due to seismic sources, Phys. Earth Planet. Inter., 94, 43-62, 1996.

Kumar, R., A. Miglani, and N. R. Garg, Plane strain problem of poroelasticity using eigenvalue approach, Proceeding Indian Acad. Sci. (Earth Planet Sci.), 109, 371-380, 2000.

Kumar, R., A. Miglani, and N. R. Garg, Response of an anisotropic liquidsaturated porous medium due to two-dimensional sources, Proceeding Indian Acad. Sci. (Earth Planet Sci.), 111, 143-151, 2002.

Love, A. E. H., A Treatise on the Mathematical Theory of Elasticity, Dover
Publications, New York, 1944.

Maruyama, T., On two-dimensional elastic dislocations in an infinite and semi-infinite medium, Bull. Earthq. Res. Inst., 44, 811-871, 1966.

Mase, G. T. and G. E. Mase, Continuum Mechanics for Engineers, CRC Press. LLC, New York, 1999.

Okada, Y., Surface deformation due to inclined shear and tensile faults in a homogeneous isotropic half space, Bull. Seismol. Soc. Am., 75, 1135$1154,1985$.

Okada, Y., Internal deformation due to shear and tensile faults in a halfspace, Bull. Seismol. Soc. Am., 82, 1018-1040, 1992.

Pan, E., Static response of a transversely isotropic and layered half space to general dislocation sources, Phys. Earth Planet. Inter, 58, 103-117, 1989.

Payton, R. G., Elastic Wave Propagation in Transversely Isotropic Media, Martinus Nijhoff Publishers, The Hague, 1983.

Saada, A. S., Elasticity - Theory and Application, Pergamon Press Inc., New York, 1974.

N. R. Garg (e-mail: nrgmath@rediffmail.com), R. Kumar, A. Goel, and A. Miglani 